\title{
30 Jahre nach dem Fall des Eisernen Vorhangs - Spannungen in Europa
}

\author{
Henning Klodt
}

Online publiziert: 20. November 2019

(C) List-Gesellschaft e.V. 2019

Die Spannungen, unter denen Europa und insbesondere die EU derzeit stehen, verleihen dem Generalthema unserer Jahrestagung vom Februar 2019 aus heutiger Sicht eine eher noch gestiegene Aktualität. Sowohl bei den Wahlen zu regionalen und nationalen Parlamenten als auch bei der Wahl zum Europäischen Parlament haben populistische Parteien fast allerorts spürbar zugelegt. Für den demokratischen Diskurs und die Konsensfindung in Europa ist das alles andere als eine gute Nachricht. Symptomatisch erscheint das Gezerre um den Brexit, das nicht nur das Vereinigte Königreich und seine parlamentarischen Institutionen zu zerreißen droht, sondern auf längere Sicht der gesamten EU ein anderes Gesicht geben könnte. Und die Bereitschaft der Nationalstaaten, sich den gemeinsamen Zielen und Werten der EU verpflichtet zu fühlen, erodiert zusehends - nicht nur, aber auch und gerade in den mittelosteuropäischen Mitgliedstaaten. Dabei konnten auf der Tagung selbstverständlich nur ausgewählte Facetten all dieser Spannungen ausgeleuchtet werden.

Den Anfang macht Michael Hüther, der mit seinem Beitrag an das Versprechen der EU erinnert, zur Konvergenz der wirtschaftlichen Entwicklung in den Mitgliedstaaten beizutragen. Er gibt eine Übersicht über die historische Entwicklung der europäischen Kohäsionspolitik (insbesondere der Regionalpolitik), über ihre theoretische Fundierung und über einige empirische Analysen dazu und gelangt daraus zu einer Bewertung dieser Politik. Die empirische Evidenz zur Entwicklung der Konvergenz in der EU zeigt allenfalls begrenzt positive Ergebnisse, bei regelmäßig geringem Einfluss der Strukturfonds. Es überwiegen allerdings die negativen Ergeb-

In diesem Heft wird berichtet über die Jahrestagung 2019 des Wirtschaftspolitischen Ausschusses im Verein für Socialpolitik, die vom 26. bis 28. Februar an der Andrássy Universität in Budapest stattfand. Ein besonderer Dank gilt Martina Eckardt und Stefan Okruch von der Andrássy Universität, die für die organisatorische Vorbereitung und Durchführung der Tagung verantwortlich waren.

H. Klodt $(\bowtie)$

Holm 19, 24107 Kiel, Deutschland

E-Mail: henning.klodt@freenet.de 
nissen - insbesondere in den Jahren seit der globalen Finanzkrise. Hüther plädiert dafür, die Kohäsionspolitik nicht mit anderen Zielsetzungen zu überfrachten und die Industriestrategie der EU auf die Schaffung angemessener Bedingungen für den volkswirtschaftlichen Strukturwandel und die Förderung der Arbeitskräftemobilität $\mathrm{zu}$ fokussieren.

Der Beitrag von Gerhard Heimpold befasst sich mit der Frage, ob der Erhalt großer ostdeutscher Industrieunternehmen (,,industrielle Kerne“) nach der Vereinigung erfolgreich gewesen ist. Das Interesse des Autors ist dabei vorrangig ein historisches, nämlich die wirtschaftswissenschaftliche Aufarbeitung der Transformationshistorie; er geht aber auch der Frage nach, ob sich die jeweiligen Standorte im globalen Wettbewerb behaupten können. Im Zentrum stehen vier Fallbeispiele, deren Entwicklung von 1990 bis heute relativ detailliert nachgezeichnet wird. Alle vier von Heimpold analysierten Kern-Unternehmen existieren bis heute fort und stellen marktgängige Produkte und Leistungen her. Die Interaktion mit dem regionalen Umfeld fällt allerdings unterschiedlich aus. Und zwar waren die positiven Wirkungen des regionalen Umfelds besonders ausgeprägt bei jenen Unternehmen, die in urbanen Räumen gelegen sind.

Ulrich Heimeshoff nimmt die europäischen Energiemärkte ins Visier. Seit Jahrzehnten stehen diese Märkte im Fokus der Europäischen Kommission, die danach strebt, die ehemals von nationalen Monopolen dominierten Märkte zu einem echten gemeinsamen Binnenmarkt zu entwickeln. Auf den von Heimeshoff näher analysierten Stromgroßhandelsmärkten herrscht zumindest in den zentral gelegenen EULändern ein hinreichender Integrationsgrad, der Wettbewerb ermöglicht. Als zentrale Messziffer dafür dient ihm das Ausmaß der regionalen Preisdifferenzierung. Für die westliche, östliche und südliche Peripherie dagegen ist der Integrationsgrad gering, wofür nach Heimeshoff unter anderem physische Engpässe bei den grenzüberschreitenden Übertragungskapazitäten verantwortlich sind. Dementsprechend lautet seine wirtschaftspolitische Handlungsempfehlung, diese Kapazitäten verstärkt auszubauen. Darüber hinaus weist er darauf hin, dass der starke Ausbau der Windkraft den Integrationsgrad der europäischen Energiemärkte tendenziell gesenkt hat. Der weitere Ausbau der erneuerbaren Energiequellen sollte demnach insbesondere dort erfolgen, wo es aus technischer und ökonomischer Sicht sinnvoll ist.

Paul Welfens beschreibt die westliche Wirtschaftspolitik seit Fall des Eisernen Vorhangs. Dabei versucht er, Fehlentwicklungen zu identifizieren, die als Konsequenz letztlich zum Vordringen des Populismus im Allgemeinen und zum Brexit im Besonderen geführt hätten. Darüber hinaus stellt Welfens Prognosen zur weiteren Entwicklung der EU und leitet daraus erforderliche Reformen ab. Bei den Fehlentwicklungen geht es unter anderem um die überzogene Liberalisierung der Finanzmärkte und die unzureichende internationale Bankenaufsicht. Eingegangen wird auch auf die zunehmende Einkommensungleichheit seit 1990 und die Expansion des Internets. Letztere habe das Systemrisiko eines Internetzusammenbruchs und eine zunehmende politische Radikalisierung durch digitale Kommunikation mit sich gebracht. Insgesamt wird in diesem Beitrag ein breites Spektrum an unterschiedlichen ökonomischen und politischen Entwicklungen und damit verbundene Problemfeldern in den vergangenen drei Jahrzehnten angerissen, die unter anderem zur Stärkung des Populismus und zum Brexit geführt hätten. 
Ivo Bischoff und seine Koautoren berichten über die Ergebnisse eines SurveyExperiments mit Studierenden in Deutschland und Polen. Die Autoren analysieren, ob politische Entscheidungen der EU auf weniger Zustimmung stoßen als vergleichbare Entscheidungen nationaler Parlamente. Als Grundlage dienen Befragungen von Studenten an drei Hochschulen in Kassel sowie in Rzeszow und Poznan in Polen. Die Ergebnisse weisen darauf hin, dass Entscheidungen mehr Zustimmung erfahren, wenn sie von nationalen Parlamenten getroffen werden und nicht von der EU (Europäisches Parlament und Europäischer Rat). Der statistisch signifikante Akzeptanzabschlag für die Entscheidungen der EU ist allerdings nicht besonders groß. Der vielfach, vor allem von EU-skeptischen Parteien beklagte, Vertrauensverlust der EU existiere somit nicht. Die Ergebnisse des Experiments ändern sich auch nicht, wenn man die Entscheidungen nach dem Subsidiaritätsprinzip vorsortiert. In der multivarianten Analyse zeigt sich, dass die Befragten die Entscheidungen der EU nicht negativer beurteilen, wenn die EU für das politische Feld nach dem Subsidiaritätsprinzip nicht zuständig sein sollte.

In dem Beitrag von Stefan Voigt werden Vorschläge zur Reform des in Artikel 7 EUV definierten Verfahrens zur Sicherung der in Artikel 2 EUV genannten Grundwerte der EU (insbesondere der Rechtsstaatlichkeit) in den Mitgliedstaaten entwickelt. Angesichts der diesen Grundwerten entgegenlaufenden politischen Entwicklungen in mehreren Mitgliedstaaten und der Unfähigkeit der EU, angemessen hierauf zu reagieren, ist diese Thematik höchst aktuell. Sie wird von Voigt aus der Perspektive der normativen Institutionenökonomik analysiert. Die von ihm vorgeschlagene Reform des Verfahrens umfasst zwei interdependente Kernelemente: die Beschleunigung des Verfahrens durch Etablierung eines „Frühwarnsystems“ sowie die Entpolitisierung des Verfahrens durch Etablierung eines Expertenkomitees und der Übertragung von Entscheidungskompetenzen von den politischen Organen der EU an dieses Expertenkomitee und an den EuGH. Dabei ist dem Autor allerdings klar, dass auf absehbare Zeit keine realistische Aussicht auf eine Umsetzung der gemachten Reformvorschläge und die dafür erforderliche Änderung der europäischen Verträge besteht.

Im abschließenden Beitrag befasst Siegfried Franke sich mit dem Vordringen sogenannter illiberaler Demokratien, wofür nicht zuletzt Ungarn ein Beispiel liefert. Als eine mögliche Ursache für diese Entwicklung verweist er auf die latent in jedem politischen System vorhandenen Versuchungen des Machterhalts und des Machtmissbrauchs. Dem stehe nach dem Rechts- und Demokratieverständnis der EU das Prinzip der Gewaltenteilung entgegen. Kritiker, wie der ungarische Regierungschef Orban, argumentieren dagegen, vom Volk durch Wahlen legitimierte Regierungen dürften nicht durch andere, schwächer legitimierte Institutionen der EU in ihrem Handeln behindert oder gebremst werden. Eine weitere Ursache für die Ausbreitung der illiberalen Demokratien liegt nach Franke darin, dass zu viele konstitutionelle Restriktionen und Mitwirkungsrechte eigennützig handelnder Dritter für Staaten im wirtschaftlichen Aufholprozess hinderlich seien. Befürworter der illiberalen Demokratie kritisieren darüber hinaus die in vielen Staaten auf die Spitze getriebenen Auswüchse der „Political Correctness“ sowie die übertriebene Toleranz auch gegenüber jenen, die aus ihrer intoleranten Haltung kein Hehl machen. Am Beispiel Ungarns erläutert Franke, wie demokratische Regierungen ein freiheitlich-rechts- 
staatliches System in eine illiberale Demokratie umwandeln können, indem sie die Gewaltenteilung aushebeln, Befugnisse autonomer Institutionen beschneiden, den Schutzbereich der Grundrechte einengen, die Aktivitäten der Zivilgesellschaft beschränken sowie Rechtsregeln und Bürokratien schaffen, denen die Bürger kaum Folge leisten können.

Insgesamt zeigen die Tagungsbeiträge in aller Deutlichkeit auf, dass die EU auch sechzig Jahre nach ihrer Gründung und dreißig Jahre nach dem Fall des Eisernen Vorhangs keineswegs in einem stabilen Gleichgewicht angekommen ist. Die hier beleuchteten Spannungen in Europa werden die Politik, die Bürger und den wirtschaftspolitischen Ausschuss im Verein für Socialpolitik aller Voraussicht nach auch künftig weiter beschäftigen.

Prof. Dr. Henning Klodt, Direktor am Institut für Weltwirtschaft a. D., Kiel. 